\title{
Multichannel Approach to Clustering Matter
}

\author{
V.I.Yukalov and E.P.Yukalova \\ International Centre of Condensed Matter Physics \\ Univesity of Brasilia, CP 04513 \\ Brasilia, DF 70919-970, Brazil
}

PACS: 05.30.-d, 05.70. Ce, 12.40 Ee, 64.10.+h

Keywords: Clustering matter, statistical models, thermodynamic functions, phase transitions 


\begin{abstract}
An approach is developed, combining the ideas of quantum statistical mechanics and multichannel theory of scattering, for treating statistical systems whose constituents can possess different bound states realized as compact clusters. The main principles for constructing multichannel cluster Hamiltonians are formulated: principle of statistical correctness, principle of cluster coexistence, and principle of potential scaling. The importance of the principle of statistical correctness is emphasized by showing that when it does not hold the behaviour of thermodynamic functions becomes essentially distorted. And moreover, unphysical instabilities can appear. The ideas are carefully illustrated by a statistical model of hot nuclear matter.
\end{abstract}




\section{Introduction}

If the constituents of a statistical system strongly interact with each other, they can form different bound states exhibiting themselves as compact clusters. Ubiquitous chemical elements are examples of such clusters [1]. Another well known example is fog consisting of droplets made of water molecules. A variety of other examples are listed in ref.[2].

Despite their so widespread prevalence there is no good theory describing statistical clustering systems. It is possible to separate three problems in this description: One is the consideration of the dynamics of growing or evaporating clusters. This process can be portrayed by complicated partial differential equations, similar to those that treat the dynamics of nuclei of a new phase or the growth and dissolution of macrodefects in nonequilibrium systems [3-5]. Another problem is the description of the properties of a single cluster inside equilibrium matter. This can be exemplified, for instance, by the model of a cluster in thermostate [2]. A fullerene molecule can also be treated as a large cluster in thermodynamically equilibrium surrounding [6,7]. A nucleon in nuclear matter can be considered as a quark bag or quark cluster $[8,9]$. The third problem is to give a statistical description of a quantum system whose particles can form various bound states, so that in the same system different types of clusters arize.

In this paper we address the third problem of the mentioned three, that is, the statistical description of a quantum system with multiple clusters. Theoretical treatment of such a system is the least understood and developed, as copmared to the first two problems, There are several objective difficulties in treating clustering matter. The main of them are as follows.

Suppose that particles forming a statistical system have various bound states. Each bound state can be described by a Bethe-Salpeter-type equation for a $z-$ particle Green function, where $z$ is the compositeness of the bound state. This type of equations, even for a two-particle Green function, is very difficult to deal with. When there are many bound states interacting with each other as well as with unbound particles, then one has to deal with a large system of many coupled Bothe-Salpeter equations for two-, three-, and so on up to the highest order $z$-particle Green functions, plus the Dyson-type equations for singleparticle Green functions [10]. Even to deal with a two-particle Green function is not as easy. And imagine that one has to operate with a ten-particle Green function, when there occur ten-particle bound states. It is evident that in the case of multiple bound states the standard approach becomes practically unsolvable and useless. Then one simplifies the problem by inventing effective thermodynamic potentials. However, because of ambiguity in constructing such potentials, the thermodynamic behaviour of the considered system may have quite different features depending on the assumptions used. Morover, the fabricated thermodynamic potential may have no relation not only to the considered clustering matter but even to any statistical system. Thus, the first question that arises is: What 
is a general criterion which any effective thermodynamical potential must satisfy for correctly representing a statistical system?

In order to simplify the problem one often postulates that solely one type of bound states can exist under the given thermodynamical parameters, say temperature and density. An ensemble of given one-type bound states then is equivalent to a thermodynamic phase. And a transformation of one phase into another goes through a genuine phase transition. Evidently, such a simplification is not merely too rough but contradicts the general properties of a quantum system in which bound and unbound states can be formed simultaneously. The latter is sinonymous to requiring that different types of clusters could coexist. The necessity of allowing for such a cluster coexistence is advocated and stressed in the present paper.

As soon as we accept the possibility of cluster coexistence, another problem, looking unsolvable, emerges. When the particles constituting a system can form several types of bound-state clusters, then it is necessary to define many interaction potentials between these clusters as well as between the clusters and the constituent particles. If the particles can make, say, ten types of clusters, then we

need to know $C_{10}^{2}+10=55$ different interaction potentials. Where the latter can be taken from?

The aim of this paper is twofold: To formulate the general principles of a correct statistical description of clustering matter and to illustrate these principles by examples.

\section{Principle of Statistical Correctness}

Wishing to analyse thermodynamic properties of a statistical system, we need to define a thermodynamic potential. Writing the latter, one usually invokes some simplifications leading to an effective thermodynamic potential $\Omega(\varphi)$ containing a set $\varphi=\left\{\varphi_{i}\right\}$ of auxiliary functions depending on space and/or thermodynamic variables. What necessary to keep in mind, first of all, is that not each effective potential can have sense, however reasonable it may look. A thermodynamic potential, to be accepted as such, must satisfy the properties formulated below.

\section{Property 1. Statistical Representability.}

An effective thermodynamic potential $\Omega(\varphi)$ represents an equilibrium statistical system if and only if it has the Gibbs form

$$
\Omega(\varphi)=\Omega[H(\varphi)]
$$

defined as

$$
\Omega[H] \equiv-T \ln \operatorname{Tr} e^{-\beta H},
$$

where $\beta \equiv T^{-1}$, and the dependence on auxiliary functions comes only through an effective Hamiltonian $H(\varphi)$. Such a thermodynamic potential is called 
statistically representable.

Thus, if one invents an effective thermodynamic potential, even pronouncing seemingly plausible words, this does not necessary mean that the invented potential describes some statistical system. If this potential is not statistically representable it corresponds to no equilibrium statistical system. For example, a thermodynamic potential in the excluded-volume approximation is not statistically representable.

\section{Property 2. Thermodynamic Equivalence.}

A statistical system defined by a Hamiltonian $H$ is thermodynamically equivalent to a system modeled by an effective Hamiltonian $H(\varphi)$ if and only if their thermodynamic potentials $\Omega$ and $\Omega(\varphi)$ are statistically representable, i.e.

$$
\Omega=\Omega[H], \quad \Omega(\varphi)=\Omega[H(\varphi)],
$$

and are equal to each other,

$$
\Omega[H]=\Omega[H(\varphi)] .
$$

The corresponding Hamiltonians are called thermodynamically equivalent.

For the case of infinite matter, condition (4) can be weakened by requiring the validity of the asymptotic, in the thermodynamic limit, equality

$$
\lim _{V \rightarrow \infty} \frac{1}{V}(\Omega[H]-\Omega[H(\varphi)])=0 .
$$

\section{Property 3. Statistical Correctness.}

The necessary condition for an effective Hamiltonian $H(\varphi)$ to be thermodynamically equivalent to a Hamiltonian $H$, not depending on auxiliary functions $\varphi$, is the variational equality

$$
\left\langle\frac{\delta}{\delta \varphi} H(\varphi)\right\rangle=0,
$$

where the variation over $\varphi$ implies the set of variations with respect to each $\varphi_{i}$, and the average of an operator $\hat{A}$ is

$$
\langle\hat{A}\rangle \equiv \frac{\operatorname{Tr} \hat{A} \exp \{-\beta H(\varphi)\}}{\operatorname{Tr} \exp \{-\beta H(\varphi)\}} .
$$

Equality (5) is an evident consequence of the property of thermodynamic equivalence. We shall call (5) the condition of statistical correctness.

Basing on these properties, we formulate the

Principle of Statistical Correctness: 
An effective thermodynamical potential is statistically correct if it is statistically representable with an effective Hamiltonian satisfying the condition of statistical correctness.

Suppose that the thermodynamic potential $\Omega=\Omega(\varphi)$ is statistically representable with the Hamiltonian

$$
H(\varphi)=\hat{E}-\sum_{i} \mu_{i} \hat{N}_{i}
$$

in which $\hat{E}$ is the energy operator, $\mu_{i}$ is a chemical potential, and $\hat{N}_{i}$ is a number-of-particle operator. If this thermodynamic potential is statistically correct, then condition (5) guarantees the validity of the thermodynamic relations

$$
\begin{gathered}
p=-\frac{\partial \Omega}{\partial V}=-\frac{\Omega}{V}, \\
\varepsilon=T \frac{\partial p}{\partial T}-p+\sum_{i} \mu_{i} \rho_{i}=\frac{1}{V}\langle\hat{E}\rangle, \\
s=\frac{\partial p}{\partial T}=\frac{1}{T}\left(\varepsilon+p-\sum_{i} \mu_{i} \rho_{i}\right), \\
\rho_{i}=\frac{\partial p}{\partial \mu_{i}}=\frac{1}{V}\left\langle\hat{N}_{i}\right\rangle
\end{gathered}
$$

for the pressure $p$, energy density $\varepsilon$, entropy density $s$, and particle density $\rho_{i}$; the volume of the system being $V$.

For the effective thermodynamic potential not satisfying condition (5) the thermodynamic relations (8) would be broken, that is, the values of the quantities on the left-hand side of (8) calculated as the corresponding derivatives or as statistical averages would be different. Such an inconsistency in (8) would signify that one should not trust to predictions derived from an effective thermodynamic potential which is statistically incorrect.

The importance of sustaining the self-consistency in the thermodynamic relations (8) for studying the thermodynamics of effective models was emphasized by Zimányi et al. [11]. Requiring the validity of (8) for an effective thermodynamic potential containing unknown correcting functions yields a complicated system of nonlinear differential equations in partial derivatives with respect to the variables $T, V$, and $\mu_{i}$. This system of equations is to be complimented by boundary conditions. Such a system has no unique solution. To extract somehow the latter, one needs several additional heuristic assumptions and fitting parameters. Contrary to this, the principle of statistical correctness formulated above is, as we shall show in the next section, much simpler to deal with and gives a unique solution.

Also, the principle of statistical correctness is more general leading to (8) but not conversely. Moreover, if one finds correcting functions directly from the 
first-order relations (8), this does not guarantee the validity of the second order relations for the specific heat

$$
C_{V}=\frac{\partial \varepsilon}{\partial T}=\frac{\beta^{2}}{V}\left(\left\langle\hat{E}^{2}\right\rangle-\langle\hat{E}\rangle^{2}\right)
$$

and the isothermic compressibility

$$
\kappa_{T}=-\frac{1}{V}\left(\frac{\partial p}{\partial V}\right)^{-1}=\frac{\beta}{\rho^{2} V}\left(\left\langle\hat{N}^{2}\right\rangle-\langle\hat{N}\rangle^{2}\right) .
$$

At the same time, the condition of statistical correctness (5) insures that (8),(9),(10) and other analogous relations of arbitrary order hold true.

\section{Principle of Cluster Coexistence}

If the particles of a quantum system form different bound states, then the latter can influence thermodynamic properties. To take this influence into account, one has to allow for the existence of these bound states, estimating their relative contribution to the properties of the system. This is equivalent to saying that it is necessary to allow for the coexistence of different clusters, calculating their statistical weights in the analyzed properties. The necessity of taking into account both unbound and various bound states seems so natural from the general quantum-mechanical point of view that it could look excessive repeating it, if it would not be so common meeting in literature statistical models in which different cluster states are prohibited to coexist, being treated as pertaining to different thermodymanic phases. Because of this frequent confusion of quantum states with statistical states we feel it is worth clarifying this question once more. A quantum bound state corresponds to a cluster but not to a thermodynamic phase. Although it may happen that under some conditions, say at low temperature, one type of clusters prevails while at high temperature another type becomes dominant, this does not mean that there is no admixture of other types of clusters among those of a predominant type. Predominance of one type of clusters is not the same as the complete prohibition to exist for other types. What types of clusters and in which proportions can coexist in a particular thermodynamic phase is, figuratively speaking, to be decided by the system itself, which implies that each system tends to a state of maximal thermodynamic stability, and the concentrations of clusters are to be defined by stability conditions. The latter can be essentially spoiled by the prohibition for clusters to coexist and can lead to incorrect thermodynamic behaviour, for instance, to the appearance of spurious phase transitions or to the change of order of genuine phase transitions.

The most general and, probably, apparent way of understanding the structure of a Hamiltonian corresponding to a system of coexisting clusters is from the 
point of view of the multichannel theory of scattering [12] considering each type of bound states, i.e., of clusters, as a reaction channel of interacting particles.

The methods of the abstract multichannel theory of scattering may be applied to physical systems of different nature, in which the constituent particles can form various bound states. Below we briefly delineate the main general ideas that could be used for any kind of such systems.

Let a system be defined by a Hamiltonian $H$ which is a selfadjoint operator acting in the Hilbert space $\mathcal{H}$, called the space of quantum states. Assume that the constituent particles of the system can form bound states. Thus, in a quarkgluon system various hadron states can be formed. Enumerate all possible types of bound states by the index $i$, where $i=1$ stands for unbound particles. Each type of bound states is individualized by a set of corresponding characteristics, such as the compositeness number $z_{i}$ showing the number of bound particles, effective mass $m_{i}$, and a set of quantum numbers like spin, isospin, colour, baryon number, and so on. All quantum states associated with the same type of bound states, indexed by $i$, compose the subspace $\mathcal{H}_{i} \subset \mathcal{H}$. In other words, $\mathcal{H}_{i}=\hat{P}_{i} \mathcal{H}$ is a projection of $\mathcal{H}$. Generally, different quantum states can be made orthogonal to each other. This means that the subspaces $\mathcal{H}_{i}$ can be considered as mutually orthogonal, that is, for the reducing projections $\hat{P}_{i}$ one has $\hat{P}_{i} \hat{P}_{j}=\delta_{i j}$. Such pairwise orthogonal projections $\hat{P}_{i}$ are called the channel projections, and the related subspaces $\mathcal{H}_{i}$ are termed the channels. A quantum state pertaining to the channel $\mathcal{H}_{i}$ is an $i$-channel state.

The set of channels $\mathcal{H}_{i}$ is complete if all possible $i$-channel states span the whole space of quantum states $\mathcal{H}$. Then the latter is written as the direct sum $\mathcal{H}=\oplus_{i} \mathcal{H}_{i}$. This is equivalent to the resolution of unity $\hat{1}=\sum_{i} \hat{P}_{i}$.

Let the time evolution of quantum states in a channel $\mathcal{H}_{i}$ be defined by a selfadjoint operator $H_{i}$. This implies that the channel $\mathcal{H}_{i}$ is invariant under the action of $H_{i}$. Because of the pairwise orthogonality of the channels, the operators $H_{i}$ are pairwise commuting, $\left[H_{i}, H_{j}\right]=0$. Each of such operators $H_{i}$ is called the channel Hamiltonian. The channel system is a system $\left\{H_{i}\right\}$ of the channel Hamiltonians, each of which acts in the corresponding channel $\mathcal{H}_{i}$. The sum $\sum_{i} H_{i}+$ const $\hat{1}$ of the channel Hamiltonians, attributed to the channel system, may be named the multichannel Hamiltonian. The latter, by construction, acts in the space of quantum states $\oplus_{i} \mathcal{H}_{i}$.

In this way, the description of an ensemble of particles forming bound states can be done by constructing the corresponding channel system. To this end, one has to classify different types of states and to define the related channel Hamiltonians $H_{i}$. The sum $\sum_{i} H_{i}+$ const $\hat{1}$ gives the Hamiltonian of the channel system. It is worth noting that the multichannel Hamiltonian does not necessarily coincide with the exact Hamiltonian $H$, but rather gives a physically reasonable first approximation. In particular calculations, one can, if necessary, resort to perturbation theory starting from the multichannel Hamiltonian. Though in many applications already the channel approximation yields quite accurate results. 
Summarizing what is said above we formulate the

\section{Principle of Cluster Coexistence:}

If the particles of an equilibrium statistical system can form clusters of different types, then all such cluster types can coexist with the probabilities defined by the condition of thermodynamic stability.

Let us concretize how one can define such channel probabilities. The multichannel Hamiltonian, in general, reads

$$
H=\sum_{i} H_{i}+C V
$$

where $H_{i}$ is an $i$-channel Hamiltonian and $C V$, a nonoperator term.

Take the channel hamiltonians, $H_{i}$, in the mean-field approximation

$$
H_{i}=\sum_{k} \omega_{i}(k) a_{i}^{\dagger}(\vec{k}) a_{i}(\vec{k})
$$

with an effective spectrum

$$
\omega_{i}(k)=K_{i}(k)+U_{i}-\mu_{i}
$$

in which $k$ is the absolute value of the momentum $\vec{k} ; K_{i}(k)$ is the kineticenergy term; $U_{i}$, a mean field; and $\mu_{i}$, the chemical potential of the $i$-type cluster. The field operator $a_{i}(\vec{k})$ is a column in the space of internal degrees of freedom, such as spin, flavor, and so on. For the mean-field Hamiltonian (12) the momentum distribution

$$
\left\langle a_{i}^{\dagger}(\vec{k}) a_{i}(\vec{k})\right\rangle=\zeta_{i} n_{i}(k)
$$

is easily calculated; $\zeta_{i}$ being a degeneracy factor, and

$$
n_{i}(k) \equiv\left[\exp \left\{\beta \omega_{i}(k) \mp 1\right\}\right]^{-1}
$$

is the Bose- or Fermi function depending on the upper or lower sign, respectively. The average density of the $i$-type clusters is

$$
\rho_{i}=\frac{\zeta_{i}}{(2 \pi)^{3}} \int n_{i}(k) d \vec{k}
$$

Note that the isotropicity of the system is assumed here, because of which $n_{i}(k)$ depends on $k \equiv|\vec{k}|$. Consequently, in (13) we could write

$$
\int n_{i}(k) d \vec{k}=4 \pi \int_{0}^{\infty} n_{i}(k) k^{2} d k
$$

but for the sake of brevity we prefer the former notation. 
The density of $i$-clusters (13) factored by the compositeness number $z_{i}$ gives the density of particles $z_{i} \rho_{i}$ that are bound in the clusters of the $i$-type. The total average density of particles is

$$
\rho=\sum_{i} z_{i} \rho_{i} .
$$

The statistical weight of each channel is characterized by the channel probability

$$
w_{i} \equiv z_{i} \frac{\rho_{i}}{\rho},
$$

of the $i$-type clusters. By definition, eq. (15) enjoys the conditions

$$
0 \leq w_{i} \leq 1, \quad \sum_{i} w_{i}=1 .
$$

The compositeness number of unbound particles, $z_{i}$, is assumed to equal one.

The chemical potentials of $i$-clusters, entering into (15), can be expressed through the given thermodynamic variables with the use of the equilibrium conditions considered. Thus, if the average density of particles (14) is given, then the $i$-cluster chemical potentials $\mu_{i}$ are related to that of unbound particles, $\mu$, by the equilibrium condition

$$
\frac{\mu_{i}}{z_{i}}=\mu \quad(\rho=\text { const }) .
$$

In the case when the average baryon density

$$
n_{B}=\sum_{i} B_{i} \rho_{i}
$$

is conserved, where $B_{i}$ is the baryon number of a cluster in an $i$-channel, then the equilibrium condition reads

$$
\frac{\mu_{i}}{B_{i}}=\mu_{B} \quad\left(n_{B}=\text { const }\right),
$$

where $\mu_{B}$ is the baryon potential of matter.

The cluster probabilities in (15), together with the kind of equilibrium considered, are defined as functions of thermodynamic variables. Finally, it is necessary to check that the considered equilibrium is stable, so that the stability conditions

$$
C_{V}>0, \quad \kappa_{T}>0
$$

for the specific heat (9) and isothermic compressibility (10) are fulfilled.

In this way, all cluster probabilities in (15) can be calculated self-consistently, thus showing the proportions in which various types of clusters are intermixed in matter. 


\section{Principle of Potential Scaling}

In order to complete the definition of a system containing different types of clusters, we need to specify the interaction potentials $\Phi_{i j}(r)$ of these clusters. If there is a number of such cluster types, then we have to define numerous potentials $\Phi_{i j}(r)$. For example, as is mentioned in Introduction, in the case of 10 cluster types we need to have 55 such potentials. And for 100 cluster types we would need already 5050 interaction potentials. Where could we take them from?

The idea of how the interaction potentials could be connected with each other comes from the form of the equilibrium conditions (17) and (19). By analogy to these conditions we may conjecture that the interaction potentials are scaled, by means of the corresponding compositeness numbers, to a universal function as follows:

$$
\frac{\Phi_{i j}(r)}{z_{i} z_{j}}=\Phi(r)
$$

Such a relation, as is clear, can be sensible only if the interaction potentials are of similar nature. To be more precise, we shall say that the interaction potentials are in the same universality class if and only is they can be scaled to one universal function, as in (21). As a counterexample we may adduce a pair of potentials one of which decreases, as $r \rightarrow \infty$, and another increases. Certainly, these potentials cannot be in the same universality class. Only the clusters of similar nature possess the interaction potentials that can pertain to one such a class.

Combining what is said above, we come to the

\section{Principle of Potential Scaling:}

The clusters of similar nature interact with each other through potentials from the universality class, so that these potentials are scaled by the corresponding compositeness numbers to a universal function.

The scaling relation (21), defining a universality class, can be derived from the following reasoning. Consider the channel reaction

$$
m+n+j \rightarrow i+j
$$

with the corresponding equation for the compositeness numbers

$$
z_{m}+z_{n}+z_{j}=z_{i}+z_{j}
$$

The reaction (22), with eq. (23), signifies that the $m$-cluster and $n$-cluster fuse together in the presence of a $j$-cluster. Assume the additivity of interactions with respect to the $j$-channel, that is,

$$
\Phi_{m j}(r)+\Phi_{n j}(r)=\Phi_{i j}(r) .
$$


Then, from (23) and (24) it is straightforward to derive (21). Therefore, the scaling relation (21) can be interpreted as the result of the additivity of interactions (24) under condition (23). Making this statement more general, but less accurate, we may say that the scaling relation (21) is a manifestation of two conservation laws: conservation of energy and conservation of particle number during the reactions of fusion and decay.

Thus, if at least one of the interaction potentials is known, say $\Phi_{m n}(r)$, then by means of the scaling relation (21), all others can be expressed through this reference potentials as

$$
\Phi_{i j}(r)=\frac{z_{i} z_{j}}{z_{m} z_{n}} \Phi_{m n}(r)
$$

This solves the problem of multiple interaction potentials making the multichannel approach to clustering matter completely defined.

\section{Clustering Nuclear Matter}

To illustrate the approach, we need to apply it to a particular clustering substance. it is possible to distinguish two quite different cases: One, when the consideration can be limited by several cluster types, say, by about ten of them; and another, when there exists a multitude of various cluster types. The latter case has to do with substances like fog which consists of droplets whose compositeness numbers range from one to many billions. A good example of the former case is nuclear matter which can be characterized by a limited number of clusters. In this way, fog is a more complicated system, which we shall consider in a separate publication. And here we analyse the more simple case of hot and dense nuclear matter, paying the main attention to the process of evaporation and condensation of hadron clusters, that is, to the deconfinement-confinement transition.

The necessity of resorting to statistical models for nuclear quark-hadron matter is due to the fact that perturbative quantum chromodynamics does not provide information on the equation of state in the whole region of thermodynamic

parameters. Especially little can be said about the most interesting region of deconfinement-confinement transition. There exist lattice calculations which, however, are reliable only for the case of zero baryon density, but not for its finite values [13].

The overwhelming majiority of statistical models for deconfinement have been based on the assumption that the latter is a phase transition between two pure phases, the clustered hadron phase and the phase of unbound quarks and gluons called the quark-gluon plasma. But this assumption contradicts to many computer simulations and also to some analytical estimates. For example, intensive numerical simulations for lattice quark-gluon plasma [14] and pure gluon plasma [15] revealed nontrivial effects due to strong particle correlations at temperatures above the deconfinement temperature $T_{d}$, which has been interpreted as the existence of hadronic modes, even at $T>T_{d}$. The deconfinement was 
found to be a rapid crossover, but not a genuine phase transition [14]. The hightemperature phase contains fluctuations being colour singlet modes, hadronic in character. This is because the poles and cuts in the linear responce functions of the hadronic phase go over smoothly into those of the high-temperature one, so that all low-temperature hadrons have analogs in the high-temperature phase. The low-and high-temperature phases both have confining characteristics, but the effect of confinement upon thermodynamic properties becomes less and less significant as temperature increases.

The confinement-deconfinement transition can be compared with the insulatormetal transition or with the ionization, since hadrons are nothing but the bound states of quarks and gluons, or small droplets of quark-gluon plasma [16-18]. In insulating solids, below the transition point to a metal, the conductivity is not strictly zero, since the ionization energy is finite and ionization can locally provide some free electrons. Similarly, in the hadron matter below the deconfinement temperature quarks can be separated. Both the conventional Mott transition in solids and the deconfinement transition in hadronic matter thus lead from a regime, in which the binding can locally be broken by ionization, to one where it is globally removed by a collective screening of the binding force.

The spatial structure of correlation functions, obtained in lattice numerical simulations for hot quark-gluon plasma, is very similar to the structure of the corresponding zero-temperature functions [14]. The thermodynamic characteristics, such as pressure and energy density, aslo display strong nonperturbative effects persisting till about $2 T_{d}$, as is found in the lattice simulations and discussed in recent surveys $[19,20]$. The fact that some lattice simulations with dynamical quarks displayed jumps of thermodynamic characteristics at $T_{d}$ can be explained [21] as merely due to the finite size of the lattice, since the transition becomes less and less abrupt as the lattice size inreases. Lengthy runs show no evidence for metastability thus suggesting that there is no sharp transition, but only a crossover phenomenon.

The coexistence scenario, based on numerical simulations, is supported also by some analytical calculations. For instance, the density-density correlation functions in the Nambu-Jona-Lasinio model contain poles and cuts that are the same at all temperatures [22]. Therefore meson modes do exist above as well as below transition temperature. Analogously, quarks and gluons should also exist in the low-temperature as well as in high-temperature phase. The graduate change in the excitation spectrum from hadronic states to quarks and gluons and the survival of hadronic modes in the high-temperature phase appear also in the magnetic-current approximation [23] and in the instanton-liquid approach [24]. This suggests the following picture of the quark-hadron coexistence. The correlations between quarks persist above the deconfinement temperature forcing some of them to correlate into colour singlets. As the quarks are moving in the heat bath, the strings connecting them for colour neutrality are constantly breaking and reforming, which can be interpreted as hadrons going in and out 
of the heat bath. This picture has a formal resemblance to the string-flip model [25] although with time-like strings.

Thus, the concept of cluster coexistence is absolutely natural from the point of veiw of the multichannel scattering theory and is supported by lattice numerical calculations. The cluster coexistence is somewhat similar, although not identical to heterophase coexistence common for many statistical systems, both these types of coexistence leading to precursor, or pretransitional, phenomena [26].

There is a variety of different statistical models trying to describe deconfinement in nuclear matter (see reviews $[27,28]$ ). The majority of these models, with a few exceptions (e.g. [29-31]), do not take into account the possible coexistence of clusters. This is why the predictions of such models are in disagreement with lattice numerical results.

Consider the clustering nuclear matter whose elementary constituents are quarks, antiquarks and gluons. These can form bound states corresponding to hadron clusters. The total set $\{i\}$ of the indices enumerating the channels can be separated into two groups, $\{i\}_{1}$ and $\{i\}_{z}$. The first group $\{i\}_{1}$ is related to unbound constituents, quarks, antiquarks, and gluons whose compositeness number $z_{i}=1$. The second group corresponds to hadron channels representing bound states with compositeness number $z_{i} \geq 2$. Respectively, the density (14) may be written as the sum

$$
\rho=\rho_{1}+\rho_{z}, \quad \rho_{1} \equiv \sum_{\{i\}_{1}} \rho_{i}, \quad \rho_{z} \equiv \sum_{\{i\}_{z}} z_{i} \rho_{i}
$$

in which $\rho_{1}$ is the density of unbound particles and $\rho_{z}$ is the density of particles in bound states.

Accept the effective Hamiltonian (11) with the channel Hamiltonians given by $(12)$. To define the effective spectrum $\omega_{i}(k)$ in $(12)$, we have to concretize the mean field $U_{i}$. The quark-gluon plasma mean field $U_{1}$ can be defined as

$$
U_{1} \equiv U(\rho)=\rho \int V(r) s(r) d \vec{r}
$$

where $V(r)$ is a confining potential and $s(r)$, a screening function. The necessity of including a screening correlation function into the mean-field approximation is the general requirement for any statistical system with nonintegrable interaction potentials [32]. The approximation (26) is the correlated Hartree approximation. The confining potential is not integrable since its mostly often used representation has the power-law behaviour

$$
V(r)=A r^{\nu} \quad(0<\nu \leq 2) .
$$

The screening function

$$
s(r)=\bar{s}\left(\frac{r}{a}\right) \quad\left(a \equiv \rho^{-1 / 3}\right)
$$


can be scaled by the mean interparticle distance $a$. Then, with the notation

$$
J^{1+\nu} \equiv 4 \pi A \int_{0}^{\infty} \bar{s}(x) x^{2+\nu} d x
$$

for the effective intensity of interactions $J$ measured in energy units, the mean field (26) becomes

$$
U(\rho)=J^{1+\nu} \rho^{-\nu / 3}
$$

The plasma mean field (29) has the following asymptotic properties:

$$
U(\rho) \rightarrow\left\{\begin{array}{cl}
\infty, & \rho \rightarrow 0 \\
0, & \rho \rightarrow \infty
\end{array}\right.
$$

The upper line here tells that quarks and gluons cannot exist as free particles outside dense nuclear matter - this is what is called the colour confinement. The lower line in (30) shows that the interparticle interaction decreases with the decrease of the average distance between particles - this is the so called phenomenon of asymptotic freedom.

The mean field for an $i$-channel corresponding to a bound hadron state can be written as

$$
U_{i}=\sum_{\{j\}_{z}} \Phi_{i j} \rho_{j}+z_{i}\left[U(\rho)-U\left(\rho_{z}\right)\right]
$$

where the summation is over the bound states, and $\rho_{z}$ is the density of particles in bound states. The first term in (31) desribes the interaction of a cluster in an $i$-channel with all other hadrons; the mean interaction potential being

$$
\Phi_{i j}=\int \Phi_{i j}(r) d \vec{r}, \quad \Phi_{i j}(r) \equiv V_{i j}(r) s_{i j}(r),
$$

where $V_{i j}(r)$ is a bare interaction potentials between the clusters of the $i-$ and $j$-types, and $s_{i j}(r)$ is a screening correlation function, so that $\Phi_{i j}(r)$ is called the screened, or effective, potential. The second term in (31), in the square brackets, models the interaction of a cluster of the $i$-channel with unbound plasma states.

Invoking the principle of potential scaling (21) for the effective potentials $\Phi_{i j}(r)$, we may write

$$
\Phi_{i j}=z_{i} z_{j} \Phi, \quad \Phi \equiv \int \Phi(r) d \vec{r}
$$

where $\Phi(r)$ is some reference function. Then (31) reduces to

$$
U_{i}=z_{i} \Phi \rho_{z}+z_{i} J^{1+\nu}\left(\rho^{-\nu / 3}-\rho_{z}^{-\nu / 3}\right) .
$$

At this point it is necessary to stress that the interaction between unbound particles and these between hadron clusters are of different nature, the former 
growing while the latter diminishing with increasing interparticle distance. This is equivalent to saying that they are from different universality classes, thus, cannot be scaled to the same reference function. Therefore, the reference function $\Phi(r)$ in (32) cannot be interpreted as an interaction potential for unbound plasma constituents. Consequently, with these two universality classes, we have to keep two parameters, $\Phi$ and $J$, which are to be chosen independently.

The Hamiltonian (11) must satisfy the principle of statistical correctness (see Sec.2). The role of auxiliary functions in (11) is played by the densities $\rho$ and $\rho_{z}$, which are functions of temperature and baryon density. Therefore, the condition of statistical correctness (5) reads

$$
\left\langle\frac{\delta H}{\delta \rho}\right\rangle=0, \quad\left\langle\frac{\delta H}{\delta \rho_{z}}\right\rangle=0
$$

Note that instead of $\rho$ and $\rho_{z}$, as auxiliary functions, we could take $\rho$ and $\rho_{1}$ or $\rho_{1}$ and $\rho_{z}$. The condition (34) does not depend on this choice, since $\rho=\rho_{1}+\rho_{z}$. Substituting (11) and (12) into (34) gives

$$
\sum_{i} \rho_{i} \frac{\delta U_{i}}{\delta \rho}+\frac{\delta C}{\delta \rho}=0, \quad \sum_{i} \rho_{i} \frac{\delta U_{i}}{\delta \rho_{z}}+\frac{\delta C}{\delta \rho_{z}}=0 .
$$

Taking account of (31) and (33) yields

$$
\frac{\delta C}{\delta \rho}=\frac{\nu}{3} J^{1+\nu} \rho^{-\nu / 3}, \quad \frac{\delta C}{\delta \rho_{z}}=-\frac{\nu}{3} J^{1+\nu} \rho_{z}^{-\nu / 3}-\Phi \rho_{z} .
$$

The solution of these equations is straightforward and, up to a constant, it is

$$
C=\frac{\nu}{3-\nu} J^{1+\nu}\left(\rho^{1-\nu / 3}-\rho_{z}^{1-\nu / 3}\right)-\frac{1}{2} \Phi \rho_{z}^{2} .
$$

In this way, the multichannel Hamiltonian (11) is completely defined. Emphasize that the term $C V$ cannot be omitted since its presence provides the validity of the principal of statistical correctness. Only retaining this term makes it possible to find the correct thermodynamic behaviour of the clustering matter.

With the Hamiltonian (11), the pressure is

$$
p=\sum_{i} p_{i}, \quad p_{i}= \pm T \frac{\zeta_{i}}{(2 \pi)^{3}} \int \ln \left[1 \pm n_{i}(k)\right] d \vec{k}
$$

and for the energy density one has

$$
\varepsilon=\sum_{i} \varepsilon_{i}, \quad \varepsilon_{i}=\frac{\zeta_{i}}{(2 \pi)^{3}} \int\left[\omega_{i}(k)+\mu\right] n_{i}(k) d \vec{k} .
$$

Taking the kinetic-energy term in the relativistic form $K_{i}(k)=\sqrt{k^{2}+m_{i}^{2}}$, where $m_{i}$ is the corresponding mass, we get the spectrum

$$
\omega_{i}(k)=\sqrt{k^{2}+m_{i}^{2}}+U_{i}-\mu_{i} .
$$


Before analysing in detail the thermodynamic behaviour of the system, let us notice that it has the following properties. In the case when $T \rightarrow 0$ and $n_{B} \rightarrow 0$, all particles are condensed into hadron clusters. And when $T \rightarrow \infty$, at any fixed $n_{B}$, only unbound states survive. Consider the high-temperature case more accurately, since in this limit we may compare the results with the available perturbative calculations in quantum chromodynamics.

When $T \rightarrow \infty$, then only quarks, antiquarks, and gluons remain, so that the set of unbound states $\{i\}_{1}$ is $\{q, \bar{q}, g\}$. The pressure (36) and energy density (37) become

$$
p \simeq \sum_{\{i\}_{1}} p_{i}, \quad \varepsilon \simeq \sum_{\{i\}_{1}} \varepsilon_{i}
$$

At temperatures much higher than the quark masses the latter can be neglected, because of which in (36) and (37) we may substitute

$$
n_{i}(k) \rightarrow\left[\exp \left\{\beta\left(k-\mu_{i}\right)\right\} \mp\right]^{-1},
$$

the chemical potentials being

$$
\mu_{q}=-\mu_{\bar{q}} \equiv \mu, \quad \mu_{g}=0
$$

Thus we come to

$$
p_{i}=\frac{\zeta_{i}}{6 \pi^{2}} \int_{0}^{\infty} \frac{k^{3} d k}{\exp \left\{\beta\left(k-\mu_{i}\right)\right\} \mp 1}, \quad \varepsilon_{i}=3 p_{i} .
$$

Take into account that the degeneracy factors of quarks and antiquarks are the same, $\zeta_{g}=\zeta_{\bar{q}}$, and that these particles are fermions while gluons are bosons. Then an exact integration yields

$$
p_{q}+p_{\bar{q}}=\frac{\zeta_{q}}{12}\left(\frac{7 \pi^{2}}{30} T^{4}+\mu^{2} T^{2}+\frac{\mu^{4}}{2 \pi^{2}}\right), \quad p_{g}=\frac{\pi^{2}}{90} \zeta_{g} T^{4} .
$$

From here, for the pressure in (39) we have

$$
p \simeq \frac{\pi^{2}}{90}\left(\frac{7}{4} \zeta_{q}+\zeta_{g}\right) T^{4}+\frac{\zeta_{q}}{12} \mu^{2} T^{2}\left(1+\frac{\mu^{2}}{2 \pi^{2} T^{2}}\right) .
$$

For the specific heat we find

$$
C_{V} \simeq \frac{2 \pi^{2}}{15}\left(\frac{7}{4} \zeta_{q}+\zeta_{g}\right) T^{3}+\frac{\mu^{2}\left(\mu^{2}-\pi^{2} T^{2}\right)}{2\left(3 \mu^{2}+\pi^{2} T^{2}\right)} \zeta_{q} T^{2}
$$

The pressure (43) is to be compared with that obtained by perturbation theory in quantum chromodynamics (see Appendix). Due to the relations

$$
\zeta_{q}=2 \times N_{f} \times N_{c}, \quad \zeta_{g}=2 \times\left(N_{c}^{2}-1\right)
$$


for the degeneracy factors of quarks and gluons, where $N_{f}$ and $N_{c}$ are the numbers of flavours and colours, respectively, we make it sure that the hightemperature pressure (43) asymptotically coincides with the perturbative pressure in $Q C D$, as $t \rightarrow \infty$.

Expression (43) can be simplfied further if we substitute there the chemical potential as a function of $T$ and $n_{B}$, which can be found from the formula for the baryon density

$$
n_{B} \simeq \frac{1}{3}\left(\rho_{q}-\rho_{\bar{q}}\right)=\frac{\zeta_{q}}{3(2 \pi)^{3}} \int\left[n_{q}(k)-n_{\bar{q}}(k)\right] d \vec{k} .
$$

With eq.(40), this gives

$$
n_{B} \simeq \frac{\zeta_{q} \mu}{18 \pi^{2}}\left(\mu^{2}+\pi^{2} T^{2}\right)
$$

From (47), we get the chemical potential

$$
\mu \simeq \frac{18 n_{B}}{\zeta_{q} T^{2}} \quad(T \rightarrow \infty)
$$

For the density of quarks and gluons we find

$$
\rho_{q} \simeq \frac{3 \zeta_{q}}{4 \pi^{2}} \zeta(3) T^{3}, \quad \rho_{g} \simeq \frac{\zeta_{g}}{\pi^{2}} \zeta(3) T^{3},
$$

where $\zeta(3)=1.20206$. Using (48) and introducing the notation

$$
\zeta \equiv \frac{7}{4} \zeta_{q}+\zeta_{g}
$$

we come to the conclusion that the high-temperature behaviour of the system asymptotically reduces to that of the Stephan-Boltzmann plasma,

$$
p \simeq p_{S B}, \quad \varepsilon \simeq \varepsilon_{S B}, \quad C_{V} \simeq C_{S B},
$$

for which

$$
p_{S B}=\frac{\pi^{2}}{90} \zeta T^{4}, \quad \varepsilon_{S B}=\frac{\pi^{2}}{30} \zeta T^{4}, \quad C_{S B}=\frac{2 \pi^{2}}{15} \zeta T^{3},
$$

with the factor (49). In what follows it will be convenient to present the results in a dimensionless form scaling them by means of the Stephan-Boltzmann expressions in (50). For the number of colours $N_{c}=3$, we have $\zeta_{q}=6 N_{f}$ and $\zeta_{g}=16$. Therefore, the factor (49) is

$$
\zeta=16\left(1+\frac{21}{32} N_{f}\right)
$$

which is to be substituted into (50). Then, e.g., the pressure becomes

$$
p_{S B}=\frac{8 \pi^{2}}{45}\left(1+\frac{21}{32} N_{f}\right) T^{4} .
$$




\section{Analysis of Thermodynamic Characteristics}

To accomplish explicit calculations of thermodynamic characteristics, we need to specify three parameters, $J, \Phi$, and $\nu$. The first of them, $J$, describes the intensity of nonperturbative interactions in the quark-gluon plasma. We can evaluate $J$ in several ways. The simplest way is to remember that nonperturbative effects in the quark-gluon plasma are commonly associated with the so-called bag constant, $B$, for which one uses different values so that $B^{1 / 4}$ lies in the interval between about 150 to $300 \mathrm{MeV}$. We can accept for $J$ the value from the middle of this interval, that is $J=225 \mathrm{MeV}$.

The constant $\Phi$, according to (32), can be chosen as $\Phi=\Phi_{33} / 9$ with $\Phi_{33}$ given by $\Phi_{33}=\int V_{33}(r) s_{33}(r) d r$, where $V_{33}(r)$ is the interaction potential between the three-quark bound states, that is between nucleons. The nucleon interaction potentials are well known from scattering experiments. There are several representations for these potentials. For nonintegrable hard-core potentials one has to take into account the correlation function $s_{33}(r)$, while for integrable soft potentials $s_{33}(r) \approx 1$. Among many known nucleon-nucleon potentials, we prefer the Bonn potential [33], which provides an accurate description of nucleon scattering and has sufficiently simple analytic form. Following the common consensus that thermodynamics of nuclear matter does not depend on the mutual orientation of spins of scattering nucleons, we average over spin directions, and assume also that the interaction between any pair of nucleons, whether these are protons or neutrons, is the same. The so-called cut-off terms of the Bonn potential can be neglected since they start playing an essential role only for very short distances $\leq 0.1 \mathrm{fm}$, which would correspond to the baryon density $n_{B} \geq 10^{3} n_{0 B}$ at which it would be hard to expect that any nucleons could survive. Here and in what follows $n_{0 B}=0.167 \mathrm{fm}^{-3}$ is the normal baryon density. The parameter $\Phi$ calculated in this way is $\Phi=35 \mathrm{MeV} \mathrm{fm}^{3}$.

The constant $\nu$ characterizes the power-law behaviour of the confining potential (27). The most frequently accepted cases are those of linear and harmonic confinement. Actually, our results do not change qualitatively for $\nu$ between these two possibilities. Let us consider, for example, the harmonic confinement. As we have checked, our results practically do not change quantitatively in the region $1.5 \leq \nu \leq 2$. Thus, in what follows $\nu \approx 2$.

Finally, we have to concretize the reaction channels that will be included into consideration. In principle, the developed approach permits us to include any number of particles. We have analysed many variants which, as we found, demonstrate similar behaviour. Not to overload this paper, here we limit ourselves by the particles listed in the Table. We included into consideration multiquark states, although their status is not yet absolutely clear, because there has been an intensive discussion about their possible presence in nuclei (see reviews [3436]) motivated by the Baldin interpretation of the commulative effect [37]. The chosen mass of the 6 -quark state corresponds to an average over the masses of 
several light dibaryons that are claimed to be observed in experiments [38]. The 9 - and 12 -quark parameters are elicited from the bag model calculations [39].

We have analysed in detail the behaviour of the main thermodynamic characteristics of the model as functions of temperature $\Theta=k_{B} T$ and relative baryon density $n_{B} / n_{0 B}$. Fig.1 shows the probability of the plasma channel

$$
w_{p l}=\frac{1}{\rho}\left(\rho_{g}+\rho_{u}+\rho_{\bar{u}}+\rho_{d}+\rho_{\bar{d}}\right) .
$$

The probabilities of hadron channels are displayed in the following figures: in Fig.2, the pion-channel probability

$$
w_{\pi}=\frac{2}{\rho}\left(\rho_{\pi^{+}}+\rho_{\pi^{-}}+\rho_{\pi^{0}}\right),
$$

in Fig.3, the summarized, excluding pions, probability of other meson channels

$$
w_{\eta \rho \omega}=\frac{2}{\rho}\left(\rho_{\eta}+\rho_{\rho^{+}}+\rho_{\rho^{-}}+\rho_{\rho^{0}}+\rho_{\omega}\right),
$$

in Fig.4, the nucleon-channel probability

$$
w_{3}=\frac{3}{\rho}\left(\rho_{n}+\rho_{\bar{n}}+\rho_{p}+\rho_{\bar{p}}\right),
$$

in Fig.5, the probability of the six-quark channel

$$
w_{6}=\frac{6}{\rho}\left(\rho_{6 q}+\rho_{6 \bar{q}}\right),
$$

and in Fig.6, the probability of the six-quarks in the Bose-condensed state. The probabilities of heavier multiquark channels are not depicted since they are negligibly small,

$$
w_{9}=\frac{9}{\rho}\left(\rho_{9 q}+\rho_{9 \bar{q}}\right)<10^{-3}, \quad w_{12}=\frac{12}{\rho}\left(\rho_{12 q}+\rho_{12 \bar{q}}\right)<10^{-5} .
$$

Figs.7-9 present some channel probabilities at zero temperature for a larger region of the relative baryon density. As is seen, the channel probabilities are continuous functions of their variables. The location of the deconfinement transition can be conventionally associated with the maxima of some of the channel probabilities.

Fig.10 demonstrates the pressure

$$
p=\sum_{i} p_{i}-C, \quad p_{i}= \pm T \frac{\zeta_{i}}{(2 \pi)^{3}} \int \ln \left[1 \pm n_{i}(k)\right] d \vec{k}
$$

and Fig.11, the energy density

$$
\varepsilon=\sum_{i} \varepsilon_{i}+C, \quad \varepsilon_{i}=\frac{\zeta_{i}}{(2 \pi)^{3}} \int \omega_{i}(k) n_{i}(k) d \vec{k}+B_{i} \mu_{B} \rho_{i} .
$$


These functions are quite monotonous but their ratio

$$
\frac{p}{\varepsilon}=c_{e f f}^{2}
$$

having the meaning of the effective sound velocity squared, exhibits in Fig.12 a maximum around $T_{d}=160 \mathrm{MeV}$. The temperature dependence of $c_{\text {eff }}^{2}$ at $n_{B}=0$ agrees with that reconstructed from lattice data.

The specific heat in Fig.13 is also monotonous, but the reduced specific heat

$$
\sigma_{V}=\frac{T}{\varepsilon} \frac{\partial \varepsilon}{\partial T}
$$

in Fig.14 again displays the maximum that can be associated with the location of the deconfinement line. The similar maximum exists for the compression modulus

$$
\kappa_{T}^{-1}=n_{B} \frac{\partial p}{\partial n_{B}}
$$

in Fig. 15.

These thermodynamic characteristics demonstrate that the transition from bound hadron states to unbound quark-gluon states is a gradual crossover. All these states are different channels of the same quantum system. Each channel is a subspace of the total Hilbert space of quantum states. Therefore, all channels, as possible states, always exist. But their statistical weights, defined by the channel probabilities, are different and change with varying thermodynamic parameters such as temperature and baryon density. The channel probabilities depend also on the Hamiltonian parameters, for instance, masses. Thus, if we take the mass of a six-quark state $m_{6}>2000 \mathrm{MeV}$, then the probability of this channel drastically drops down. Generally, among the members of the same family of states only those with lower masses are the most important. The states with heavier masses have, as a rule, much smaller statistical weights.

Note also that the temperature behaviour of thermodynamic characteristics at nonzero baryon density is modified as compared to that at zero baryon density: Increasing $n_{B}$ smoothes the transition. Therefore one has to be quite cautious about those predictions for the processes at nonzero baryon density, which have been made basing on the processes at $n_{B}=0$.

In conclusion, we would like to emphasize that the main in this paper are not particular numerical predictions, though, we think, they are quite reasonable, but rather the whole picture and the ideology which the latter is based on. The basis of the model suggested rests upon the multichannel interpretation of different bound states and on the principle of statistical correctness permitting us to construct a correct multichannel Hamiltonian.

Within the framework of this approach many details can be changed and some improvements may be made. For example, instead of the Bonn potential we could opt for some other effective nucleon-nucleon interactions, we could include more 
kinds of bound states, and so on. Also, we have not discussed here the role of strangeness whose generation can be important as a diagnostic tool for the study of the quark-gluon plasma signals in realistic nuclear collisions [40]. The work on these problems is under progress. Changing particular details of the model might slightly alter some quantitative values, but the qualitative picture should remain the same. According to this picture, the deconfinement is a gradual crossover from hadron states to the quark-gluon plasma in agreement with the lattice numerical calculations [14].

\section{Discussion}

In this paper we suggested a general approach for treating complex systems whose constituent particles can form different bound states realized as compact clusters. The approach is based on three main notions: principle of statistical correctness, principle of cluster coexistence, and principle of potential scaling. The last two principles are compulsory for a self-consistent treatment of clustering matter. The disregard of the first principle, that of statistical correctness, can sometimes be admissible when one is looking for a very rough approximate picture of clustering. However, if one wishes to get a really accurate description, the principle of statistical correctness must be satisfied. Moreover, neglecting this principle leads in some cases to the appearance of unphysical instabilities around phase transition points [28].

To illustrate the importance of using the princliple of statistical correctness, let us consider a simple clustering system consisting of two species, of unbound gluons and of glueballs that are bound gluon clusters. Include into consideration five lightest glueballs with the masses found in the bag-model calculations [41]. Take into account that the gluon as well as glueball chemical potentials are zero, $\mu_{i}=0$. Consider, for concreteness, the system with the $S U(2)$ symmetry when $N_{c}=2$ and the gluon degeneracy factor is $\zeta_{g}=6$. And let us calculate thermodynamic characteristics of such a system for two cases: when the principle of statistical correctness is satisfied and when it does not. In the second case we simply put zero the correcting term $C V$ in the Hamiltonian (11). The results of calculations are presented in figs.16-21, where the specific heat, pressure, and energy density are given in relative units, with respect to the corresponding quantities of the quarkless Stefan-Boltzmann plasma. The relative energy density and pressure are compared with the lattice numerical data, fitting the parameters of the model so that to have deconfinement at $T_{d} \cong 220 \mathrm{MeV}$. The qualitative behaviour of thermodynamic characteristics is similar in both cases, in the presence of the correcting term $C V$ and without it. At low temperatures, practically all gluons are clustered into glueballs. The glueball channel probability $w_{G}$ drastically drops down at the deconfinement temperature (fig.16). The gluon channel probability $w_{g}$ behaves oppositely to $w_{G}$, as is shown in fig.17. The deconfine- 
ment is a second order transition with a sharp peak of specific heat (fig.18). The comparison of the relative energy density with lattice numerical data for the case of the model without the correcting term is given in fig.19, as compared with the data of ref.[42], and in fig.20, as compared with those from ref.[43]. The relative energy density and pressure for the model satisfying the principle of statistical correctness are presented in fig.21, compared with the lattice numerical data [43]. As is clearly seen from these comparisons, the model without the correcting term does not fit well the numerical data, while the statistically correct model is in perfect agreement with the latter.

\section{Appendix. High-Temperature Limit}

In section 5 we considered the high-temperature behaviour of pressure stating that it coincides with the high-temperature asymptotic expression for pressure in quantum chromodymanics. Here we show this explicitly.

The effective coupling parameter $g=g(T)$ in quantum chromodynamics is given by the equation

$$
g^{2}(T) \simeq \frac{24 \pi^{2}}{\left(11 N_{c}-2 N_{f}\right) \ln (T / \Lambda)},
$$

where $N_{f}$ is the number of flavours and $\Lambda$ is a scaling parameter, $\Lambda \approx$ $200 \mathrm{MeV}$. The coupling $g(T) \rightarrow 0$ as $T \rightarrow \infty$. Therefore, perturbation theory in powers of $g$ becomes admissible. Perturbative expansions for the free energy have been obtained $[44,45]$ to order $g^{5}$. The fourth and fifth orders of these expansions contain an arbitrary renormalization scale. The $Q C D$ pressure can be written [17] as

$$
p=A T^{4}
$$

where the factor $A$, as $T \rightarrow \infty$, is

$$
A \simeq A_{0}+A_{2} g^{2}+A_{3} g^{3}
$$

with the coefficients

$$
\begin{gathered}
A_{0}=\frac{\pi^{2}}{45}\left[N_{c}^{2}-1+\frac{7}{4} N_{c} N_{f}+15 N_{c} N_{f} \frac{\mu^{2}}{2 \pi^{2} T^{2}}\left(1+\frac{\mu^{2}}{2 \pi^{2} T^{2}}\right)\right], \\
A_{2}=-\frac{N_{c}^{2}-1}{144}\left[N_{c}+\frac{5}{4} N_{f}+9 N_{f} \frac{\mu^{2}}{2 \pi^{2} T^{2}}\left(1+\frac{\mu^{2}}{2 \pi^{2} T^{2}}\right)\right], \\
A_{3}=\frac{N_{c}^{2}-1}{12 \pi}\left(\frac{1}{3} N_{c}+\frac{1}{6} N_{f}+\frac{1}{3} N_{f} \frac{\mu^{2}}{2 \pi^{2} T^{2}}\right)^{3 / 2} ;
\end{gathered}
$$

the chemical potentials of quarks are assumed to be equal to $\mu$. When $g \rightarrow 0$, only the term $A_{0}$ remains. The pressure $p \simeq A_{0} T^{4}$, with the relations in (45), coincides with expression (43). 
In the particular case of $N_{c}=3$, and taking into account that $\mu \rightarrow 0$ as $T \rightarrow \infty$, we have

$$
\begin{aligned}
& A_{0}=\frac{8 \pi^{2}}{45}\left(1+\frac{21}{32} N_{f}\right), \\
& A_{2}=-\frac{1}{6}\left(1+\frac{5}{12} N_{f}\right), \\
& A_{3}=\frac{2}{3 \pi}\left(1+\frac{1}{6} N_{f}\right)^{3 / 2} .
\end{aligned}
$$

Comparing the pressure $p=A T^{4}$ with the Stphan-Boltzmann limit from the end of section 5 , we get

$$
\frac{p}{p_{S B}} \simeq 1+a_{2} g^{2}+a_{3} g^{3}
$$

where

$$
a_{2} \equiv \frac{A_{2}}{A_{0}}, \quad a_{3} \equiv \frac{A_{3}}{A_{0}} .
$$

Thus, as $g \rightarrow 0$, the pressure tends to the Stephan-Boltzmann form. 


\section{References}

[1] D. ter Haar, Elements of Statistical Mechanics (Butterworth-Heinemann, Oxford, 1995).

[2] V.I. Yukalov and E.P. Yukalova, Physica A 223 (1996) 15.

[3] V.V. Slezov and P.N. Ostapchuk, Fiz. Tverd. Tela 32 (1990) 521.

[4] V.V. Slezov, Fiz. Tverd. Tela 33 (1991) 1846.

[5] V.V. Slezov, Fiz. Tverd. Tela 33 (1991) 2640.

[6] V.I. Zubov, N.P. Tretiakov, J.N. Teixeira Rabelo and J.F. Sanchez Ortiz, Phys. Lett. A 194 (1994) 223.

[7] V.I. Zubov, N.P. Tretiakov, J.F. Sanchez and A.A. Caparica, Phys. Rev. B 53 (1996) 12080.

[8] G.G. Bunatyan, Yad. Fiz. 43 (1986) 294.

[9] G.G. Bunatyan, Yad. Fiz. 51 (1990) 1243.

[10] G. Röpke, D. Blaschke and H. Schulz, Phys. Rev. D 38 (1988) 3589.

[11] J. Zimányi, B. Lukás, P.Lévai, J. Bondorf and N. Balazs, Nucl. Phys. A 484 (1988) 647.

[12] H. Baumgärtel and M. Wollenberg, Mathematical Scattering Theory (Birkhäuser, Basel, 1983).

[13] M.P. Lombardo, J.B. Kogut and D.K. Sinclair, Phys. Rev. D 54 (1996) 2303.

[14] C. Bernard, T. Blum, C. DeTar, S. Gottlieb, K. Rummukainen, U. Heller, J. Hetrick, D. Toussaint and R. Sugar, Phys. Rev. D 54 (1996) 4585.

[15] T.A. DeGrand and C.E. DeTar, Phys. Rev. D 35 (1987) 742.

[16] D.J. Gross, R.D. Pisarski and L.G. Yaffe, Rev. Mod. Phys. 53 (1981) 43.

[17] E.V. Shuryak, Phys. Rep. 115 (1984) 151.

[18] L. McLerran, Rev. Mod. Phys. 58 (1986) 1021.

[19] J. Engels, F. Karsh and K. Redlich, Nucl. Phys. B 435 (1995) 295.

[20] D.H. Rischke, Nucl. Phys. A 583 (1995) 663.

[21] J.B. Kogut, D.K. Sinclair and K.C. Wang, Phys. Lett. B 263 (1991) 101. 
[22] T. Hatsuda and T. Kunihiro, Phys. Lett. B 185 (1987) 304.

[23] C. Adami and G.E. Brown, Phys. Rep. 234 (1993) 1.

[24] T. Schäfer and E.V. Shuryak, Phys. Lett. B 356 (1995) 147.

[25] S. Gardner, C. Horowitz and J. Piekarewicz, Phys. Rev. C 50 (1994) 1137.

[26] V.I. Yukalov, Phys. Rep. 208 (1991) 395.

[27] R. Hagedorn, Riv. Nuovo Cimento 6 (1983) 1.

[28] V.I.Yukalov and E.P. Yukalova, Phys. Part. Nucl. 28 (1997) 89.

[29] V.I. Yukalov, E.P. Kadantseva and A.A. Shanenko, Nuovo Cimento A 105 (1992) 371.

[30] A.A. Shanenko, E.P. Yukalova and V.I. Yukalov Nuovo Cimento A 106 (1993) 1269.

[31] A.A. Shanenko, E.P. Yukalova and V.I. Yukalov, Phys. Dokl. 40 (1995) 291.

[32] V.I. Yukalov, Phys. Rev. A 42 (1990) 3324.

[33] R. Mchleidt, K. Holinde and C. Elster, Phys. Rep. 149 (1987) 1.

[34] J.P. Vary, Nucl. Phys. A 418 (1984) 195.

[35] V.V. Burov, V.K. Lukyanov and A.I. Titov, Phys. Part. Nucl. 15 (1984) 1249 .

[36] B.L. Bakker and I.M. Narodetskii, Adv. Nucl. Phys. 21 (1994) 1.

[37] A.M. Baldin, Phys. Part. Nucl. 8 (1977) 429.

[38] Y.A. Troian, A.V. Nikitin, V.N. Pechenov, Y.D. Beznogikh, A.G. Doroshenko and A.P. Tsarenkov, Yad. Fiz. 54 (1991) 1301.

[39] V.A. Matveev and P. Sorba, Nuovo Cimento A 45 (1978) 257.

[40] B. Müller, Rep. Prog. Phys. 58 (1995) 611.

[41] J. Donoghue, K. Johnson and B. Li, Phys. Lett. B 99 (1981) 416.

[42] J. Engels, F. Karsch, I. Montvay and H. Satz, Phys. Lett. B 101 (1981) 89.

[43] J. Engels, J. Finberg, K. Redlich, H. Satz and M. Weber, Z. Phys. C 42 (1989) 341.

[44] C. Zhai and B. Kastening, Phys. Rev. D 52 (1995) 7232.

[45] E. Braaten and A. Nieto, Phys. Rev. D 53 (1996) 3421. 


\section{Figure Captions}

\section{Fig.1}

The probability of the quark-gluon plasma channel as a function of temperature $\Theta=k_{B} T$ in $M e V$ and of the relative baryon density $n_{B} / n_{0 B}$.

\section{Fig.2}

The $\pi$-meson channel probability on the temperature-baryon density plane.

\section{Fig.3}

The summary probability of the $\eta-, \rho-$, and $\omega$-meson channels.

\section{Fig.4}

The nucleon channel probability.

\section{Fig.5}

The six-quark channel probability.

\section{Fig.6}

The channel probability of six-quarks in the Bose-condensed state.

\section{Fig.7}

The quark-gluon plasma channel probability at zero temperature as a function of the relative baryon density.

\section{Fig.8}

The nucleon channel probability at zero temperature vs. the relative baryon density.

\section{Fig.9}

The six-quark channel probability at zero temperature vs. the relative baryon density.

\section{Fig.10}

The pressure (in units of $J^{4}$ ) of the multichannel model on the temperaturebaryon density plane.

\section{Fig.11}

The energy density (in units of $J^{4}$ ) of the multichannel model.

\section{Fig.12}

The pressure-to-energy density ratio of the multichannel model. 


\section{Fig.13}

The specific heat (in units of $J^{3}$ ) for the multichannel model.

\section{Fig.14}

The reduced specific heat of the multichannel model.

\section{Fig.15}

The compression modulus (in units of $J^{4}$ ) of the multichannel model.

\section{Fig.16}

The glueball channel probability as a function of temperature.

\section{Fig.17}

Comparison of the glueball and gluon channel probabilities.

\section{Fig.18}

Reduced specific heat for the gluon-glueball mixture.

\section{Fig.19}

Relative energy density for the gluon-glueball model without the correcting term (solid line), as compared with the lattice Monte Carlo data [42].

\section{Fig.20}

Relative energy density for the gluon-glueball model without the correcting term (solid line) compared with the lattice numerical simulations [43].

\section{Fig.21}

Relative energy density and pressure for the gluon-glueball model satisfying the principle of statistical correctness (solid line) as compared with the lattice numerical data [43]. 


\section{Table Caption}

The parameters of the particles included into the numerical investigation of thermodynamics of the multichannel model. 